\section{OPEN ACCESS}

Edited by:

Jay S. Pearlman,

Institute of Electrical and Electronics

Engineers, France

Reviewed by:

Frank Edgar Muller-Karger,

University of South Florida,

United States

Laurent Coppola,

UMR7093 Laboratoire

d'océanographie de Villefranche

(LOV), France

*Correspondence:

Rachel Przeslawski rachel.przeslawski@ga.gov.au

Specialty section: This article was submitted to

Ocean Observation,

a section of the journal Frontiers in Marine Science

Received: 19 November 2018 Accepted: 20 March 2019

Published: 05 April 2019

Citation:

Przeslawski R, Foster S, Monk J, Barrett N, Bouchet P, Carroll A,

Langlois $T$, Lucieer $V$, Williams $J$ and Bax N (2019) A Suite of Field Manuals for Marine Sampling to Monitor Australian Waters.

Front. Mar. Sci. 6:177. doi: 10.3389/fmars.2019.00177

\title{
A Suite of Field Manuals for Marine Sampling to Monitor Australian Waters
}

Rachel Przeslawski ${ }^{\star *}$, Scott Foster ${ }^{2}$, Jacquomo Monk ${ }^{3}$, Neville Barrett ${ }^{3}$, Phil Bouchet ${ }^{4,5}$,
Andrew Carroll' ${ }^{1}$,im Langlois ${ }^{4}$, Vanessa Lucieer ${ }^{3}$, Joel Williams ${ }^{6}$ and Nicholas Bax ${ }^{2,7}$

${ }^{1}$ National Earth and Marine Observations Branch, Geoscience Australia, Canberra, ACT, Australia, ${ }^{2}$ CSIRO Data61, Hobart, TAS, Australia, ${ }^{3}$ Institute for Marine and Antarctic Studies, University of Tasmania, Hobart, TAS, Australia, ${ }^{4}$ School of Biological Sciences, UWA Oceans Institute, The University of Western Australia, Crawley, WA, Australia, ${ }^{5}$ School of Ocean Sciences, Bangor University, Bangor, United Kingdom, ${ }^{6}$ NSW Department of Primary Industries, Taylors Beach, NSW, Australia, ${ }^{7}$ CSIRO Oceans and Atmosphere, Hobart, TAS, Australia

One of the main challenges in assessing marine biodiversity is the lack of consistent approaches to monitor it. This threatens to undermine ocean best practice in marine monitoring, as it impedes a reduction in the bias and variance of sampled data and restricts the confidence in the advice that can be given. In particular, there is potential for confounding between the monitoring methods, their measured ecological properties, and the questions they seek to answer. Australia has developed significant longterm marine monitoring and observing programs and has one of the largest marine estates, including the world's largest representative network of marine parks. This new network will require ongoing monitoring and evaluation, beyond what direct funding can support, which needs to be integrated in a standardized way with other national programs to develop sufficient monitoring capacity. The aim of this paper is to describe the process undertaken in developing a suite of field manuals that provide Standard Operating Procedures (SOPs) for marine sampling in Australian waters so that data are comparable over time and space, thereby supporting a robust, cost-effective, and objective national monitoring program. We encourage readers to refer to the complete manuals of interest at www.nespmarine.edu.au/field-manuals. We generally limit SOP development to benthic or demersal sampling, (multibeam, autonomous underwater vehicles, baited remoted underwater video (BRUV), towed imagery, grabs and box corers, sleds and trawls), with a few exceptions (e.g., pelagic BRUVs). Collaboration was a key characteristic of our approach so rather than single groups trying to impose their standards, more than 70 individuals from over 30 organizations contributed to the first version of this field manual package. We also discuss the challenges that arose while developing these national SOPs, the associated solutions that were implemented, and the plans for ensuring their long-term maintenance and national and international uptake. We anticipate that this paper will contribute to international collaborations by evoking valuable suggestions and sharing of lessons learnt from other national initiatives so that we might work toward a global ocean best practice for biological and geoscientific monitoring of the marine environment.

Keywords: multibeam, marine imaging, grab, box corer, epibenthic sled, autonomous under water vehicle, survey design, marine monitoring 


\section{INTRODUCTION}

Australia has the world's third largest marine estate, characterized by vulnerable habitats with high biodiversity and a variety of endemic species extending across a wide latitudinal range (Butler et al., 2010). This marine estate is used by a range of industries including fisheries, energy, and shipping (Dunstan and Dambacher, 2019), in addition to traditional, cultural, scientific and recreational uses. Monitoring the impacts of human activities throughout this vast ocean space represents a massive shared responsibility that can only be achieved by making the best use of all available empirical information. The Commonwealth Government has recently established the Australian Marine Parks (AMPs), including 60 marine parks managed through six regional plans. This is the largest representative network of marine parks in the world, complementing existing networks in State and Territory waters (Cochrane, 2016). Australia now has a number of successful long-term marine monitoring and observing programs (e.g., GBRMPA, 2015; Stuart-Smith et al., 2017). There is also a national integrated marine observing system (Lara-Lopez et al., 2016) that incorporates an ocean data network ${ }^{1}$ with well-established monitoring networks for the physical ocean that meet international standards and a desire to increase its biological coverage with comparable rigor (Boss et al., 2018). Without common and agreed standards for collecting marine data, the information collected will not be comparable between survey times either in the one area, or at the same time across multiple areas (Costello et al., 2017). This will reduce the value of such data over the short- and long-term for both regional and national management initiatives, marine asset inventory assessment and policy development, thereby ultimately reducing the relevance of science to decision makers and funders and vice versa.

Marine sampling standards have been adopted at a national scale by researchers for marine imagery classification (Althaus et al., 2015) and acoustic tracking of pelagic animals (Hoenner et al., 2018), but this had not been done on a national scale for most biological and geoscientific sampling platforms prior to the current program. A plan was therefore designed to develop, test, implement and adopt Standard Operating Procedures (SOPs) for monitoring in Australian marine waters, facilitated through an Australian Government funded national marine science collaboration ${ }^{2}$.

One of the main challenges in assessing marine biodiversity is the lack of standardized approaches for monitoring (Duffy et al., 2013; Teixeira et al., 2016). Although not all sampling platforms are comparable, the overarching goal of ocean best practice in marine monitoring is to reduce the bias and variance in inference from sampled data and to reduce potential confounding that can be introduced with different sampling procedures (Albert et al., 2010). This ensures that inferred trends reflect true underlying processes at play within the observed community rather than sampling artifacts (Monk, 2014; Bates et al., 2015). If measured ecological variables (e.g., abundance,

${ }^{1}$ www.aodn.org.au

${ }^{2}$ www.nespmarine.edu.au diversity, and size) and the variation in sampling techniques are confounded, it is challenging, if not impossible, to objectively determine if observed changes are due to real ecological change or sampling technique (e.g., Goddard and Foster, 2002). If variability between sampling techniques is sufficiently high, real changes that would trigger appropriate management responses may not be detected, incorrect advice might be provided to decision-makers and the influence and opportunities for marine science, especially monitoring is reduced. The lack of effective marine environmental monitoring is recognized as a significant limitation to achieving progress again United Nations Sustainable Development Goal 14 (Oldekop et al., 2016).

The aim of this paper is to describe the process undertaken to develop a suite of field manuals that present SOPs for marine sampling so that data are comparable in time and through space, thereby supporting a robust and valuable national monitoring program. The SOPs are comprehensive rather than prescriptive, monitoring methods do not have to identical, but they do need to be sufficiently well documented that process can be distinguished from trends during analysis, especially metaanalyses. We encourage readers to refer to the full content of the manuals at www.nespmarine.edu.au/field-manuals, as this paper focuses on the challenges that arose while developing national SOPs, the associated solutions that were implemented, and the plans for ensuring their long-term maintenance and uptake. We anticipate that this paper will contribute to international collaborations by evoking valuable suggestions and sharing of lessons learnt from other national initiatives so that we might work toward a global ocean best practice for geoscientific and biological monitoring.

\section{DEVELOPMENT}

All field manuals followed the same general process of development which included three discrete stages: (i) identify the target sampling platforms, (ii) generate the content for each specific platform, (iii) and review and revise.

\section{Identify Sampling Platforms}

We identified preliminary sampling platforms for the field manuals based on their frequency of use in previous sampling and monitoring programs. An online questionnaire was used to canvas the Australian marine science community on their frequency of use and perceptions regarding each of the platforms (results presented in Przeslawski et al., 2018b). We used this information to identify seven marine sampling platforms for which we would develop SOPs (Figure 1). The developed SOPs represent a range of data targets and environments (Table 1), and each has inherent advantages and limitations (Table 2). We generally limited SOP development to benthic or demersal biological sampling, with a few exceptions [e.g., pelagic baited remote underwater video (BRUV) included as a proof-of-concept due to its similarity to benthic BRUV; water column, sedimentology, and geochemistry data included for comprehensiveness related to grabs and box corers]. During this process, it became clear that a separate manual on marine 


\section{Field Manual Package}

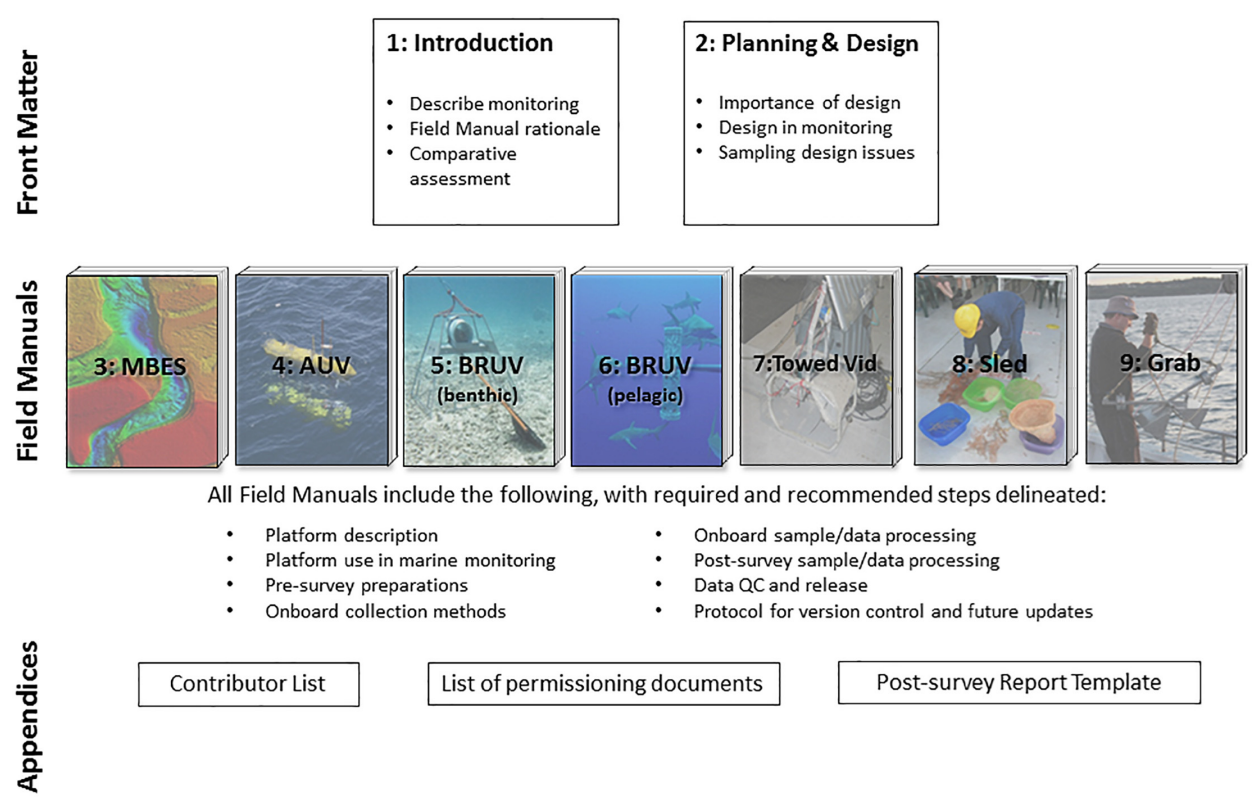

FIGURE 1 | The format of Field Manuals for Marine Sampling to Monitor Australia's Waters, from Przeslawski and Foster (2018). Numbers indicate chapters, with chapters 2-9 representing standalone manuals that present SOPs.

survey design was crucial to ensure that resulting data are appropriate for the research question, representative to facilitate population-level generalizations, and information-rich to reduce uncertainty (Figure 1).

\section{Generate Content}

In recognition of the multiple disciplines captured by the field manuals, we compartmentalized the project by allocating working group coordinators to each field manual. Working group coordinators had extensive field and analytical experience related to their respective platforms or topics and were therefore wellplaced to invite potential collaborators and consider disparate opinions. Coordinators were responsible for forming their associated working group, developing content with this group, and liaising with the editors to keep the field manual within scope and on time. Using this approach, we made sure that each chapter was written in an appropriate style for the target audience and contained the relevant information for performing the specific sampling tasks.

The Marine Sampling Field Manuals do not mandate the use of a particular sampling platform, supporting previous recognition that a top-down, one-size-fits-all approach to monitoring is unlikely to be effective in systems with large environmental variability (Fancy et al., 2009; Woodall et al., 2018). For a comparative assessment of benthic and pelagic sampling platforms, we refer readers to Przeslawski et al. (2018a); Bouchet et al. (2018), respectively. Rather, the field manuals focus on data acquisition and post-processing including data management (including quality control and post-processing), particularly as applied to marine monitoring. Standardization of sampling designs is also important and is accordingly addressed as a separate chapter. Data analysis and reporting are generally not included in the field manuals, although we direct users to useful methods or resources within each manual.

TABLE 1 | Summary of prioritized benthic sampling platforms and their acquisition targets, from Przeslawski and Foster (2018).

\begin{tabular}{lllll}
\hline & Data type & Data target & Spatial coverage & Environment \\
\hline MBES & Bathymetry, backscatter & Seafloor & Continuous & All \\
AUV & Imagery & Epibiota $^{1}$ & Continuous & All \\
BRUV & Imagery & Demersal and Pelagic fish ${ }^{1}$ & Point/transect (qualitative) & All \\
Towed & Imagery & Epibiota ${ }^{1}$ & Transect & All \\
Grab/Boxcore & Biological and sediment samples & Macrofauna, infauna, sediments & Point & Unconsolidated substrate \\
Sled/Trawl & Biological and sediment samples & Megafauna, epifauna & Transect (qualitative) & Consolidated substrate \\
\hline
\end{tabular}

MBES, multibeam echosounder; AUV, autonomous underwater vehicle; BRUV, baited remote underwater video. ${ }^{1}$ Substrate, relief, and habitat information can also be extracted from imagery, but this is usually a secondary data target. 
TABLE 2 | Advantages of prioritized benthic sampling platforms, from Przeslawski and Foster (2018).

\begin{tabular}{|c|c|c|c|c|c|c|}
\hline & MBES & AUV & BRUV & Towed & Grab/Boxcore & Sled/Trawl \\
\hline Continuous (i.e., grid) broad-scale spatial coverage & $x$ & & & & & \\
\hline Continuous (i.e., grid) fine-scale spatial coverage & & $x$ & & & & \\
\hline Non-extractive & $x$ & $x$ & $x$ & $x$ & & \\
\hline Able to revisit exact location (repeatability) & $x$ & $x$ & & & & \\
\hline Able to sample over variety of environments & $x$ & $x$ & $x$ & $x$ & & \\
\hline Species-level identifications ${ }^{1}$ & & & & & $x$ & $x$ \\
\hline Genetic, morphological etc., analysis possible & & & & & $x$ & $x$ \\
\hline Behavior observed & & & $x$ & $x$ & & \\
\hline Cryptofauna included & & & & & $x$ & $x$ \\
\hline Quantitative & $x$ & $x$ & $x$ & $x$ & $x$ & \\
\hline Concurrent physical and biological data & & $x$ & & $x$ & $x$ & \\
\hline Minimal technical expertise & & & $x$ & $x$ & $x$ & $x$ \\
\hline Vessel flexibility & & & $x$ & $x$ & $x$ & \\
\hline
\end{tabular}

${ }^{1}$ Refers to identifications able to be made with unknown or cryptic species (i.e., well-known, distinctive species can be identified via imagery).

Overall, these field manuals are meant to cover the foundational steps and key considerations, with agency- and gear-specific SOPs supplemented as needed by individual researchers. Detailed and gear-specific SOPs are outside the scope of this field manual package due to the large number of existing SOPs and the variety of gear currently employed by researchers. In this first version of the field manuals, it is impractical that researchers would agree on detailed SOPs (and associated gear). Rather, we developed the manuals with the intension of reaching a consensus about as many issues as possible, while noting discrepancies. The reporting requirements for using the SOPs record the individual differences of the approaches so that they can then be assessed in future analyses, and issues can then be addressed when they contribute to large amounts of variation in the data. Wherever possible, however, we have mandated or recommended specifications (e.g., imagery resolution) that should be used in future equipment upgrades or purchases.

Further summaries of the content of each manual are given in the Supplementary Material.

\section{Review and Revise}

The editors and working group coordinators managed the review process, which required at least one external reviewer for each chapter, as well as several others who assessed the entire package. In some cases, reviewers voluntarily provided such extensive contributions that they will be included as co-authors in the next version of the field manuals.

Ultimately, over 70 individuals from more than 30 organizations contributed to the field manual package. By engaging researchers, managers, and technicians from multiple agencies with a variety of experience, sea time, and subject matter expertise, we strove to ensure the field manuals represented an accurate and comprehensive cross-section of the broader marine science community of Australia. This not only improved the content but also increased the potential for adoption of the SOPs across multiple agencies and monitoring programs. Further details on the development of the field manuals are discussed below as they relate to particular challenges.

\section{CHALLENGES}

\section{Establishing Scope}

Establishing, justifying and communicating the scope of the field manuals were essential to expedite development, manage expectations, and distinguish from previous SOPs. The initial project scope included benthic biological sampling platforms relevant to monitoring. During content development, detailed and gear-specific SOPs (e.g., Smith MacIntyre grab, brand name systems) were assessed for inclusion in the field manuals. Ultimately, these were determined to be outside the scope of this field manual package due to the large number of existing SOPs and the variety of equipment currently employed by researchers. It was deemed impractical that researchers would agree on detailed SOPs (and associated gear) in this first version of the field manuals. Rather, we strove to reach a consensus about as many aspects as possible, while noting the differences due to agencyand gear-specific needs. These differences can then be assessed in the future (e.g., they may not correspond to large amounts of variation in data), and addressed if need be. Wherever possible, however, we have mandated or recommended specifications (e.g., imagery resolution) that should be used in future equipment upgrades or purchases.

\section{Reaching Consensus}

One of the strengths of these field manuals is the large number of collaborators involved, but this also proved to be one of the greatest challenges regarding reaching a consensus. The content development stage included ample opportunities for dissenting views to be presented to the whole group, with subsequent discussion and debate encouraged. These open forums ensured that the working group clearly justified the methods that were eventually advocated in the field manuals. In the few cases where agreement did not occur, the decisions were made by the working group coordinator and editors, who again provided a rationale for their choice.

The review process also proved valuable in reaching consensus. Reviewers noted when they disagreed with a 
recommendation or didn't understand the underlying rationale. In these cases, the lead author or editors were able to take these comments back to the working group to further refine the SOPs or associated text in the field manuals.

\section{Promoting Data Discoverability and Accessibility}

All field manuals, excluding the manual on survey design, include a section titled "Data Release," which describes ways to ensure public discoverability and accessibility of collected data, thereby abiding by the FAIR (findable, accessible, interoperable, reusable) principles (Wilkinson et al., 2016). We recognize that in the first version these sections do not provide a clear national standard and instead refer to anticipated improvements in subsequent versions. This vagueness is due to the current lack of established national data infrastructure able to incorporate appropriate or comprehensive information produced from the sampling platforms. For example, Squidle $+^{3}$ and GlobalArchive ${ }^{4}$ are currently recommended in the field manuals as the national platforms to ensure discoverability and accessibility of marine imagery, but the platforms currently have no long-term support and other platforms are being used that have potential to achieve similar discoverability and accessibility goals (e.g., Benthobox ${ }^{5}$. Similarly, species occurrence data from sledcollected specimens eventually appears on the Atlas of Living Australia $^{6}$ or Ocean Biogeographic Information System ${ }^{7}$, but this is an often circuitous route through museums and very rarely includes species absence or sampling effort information crucial for ecological and monitoring purposes (but see Coro et al., 2016 for a statistical method to estimate marine species absences from OBIS).

To meet these challenges related to data discoverability and accessibility, a series of workshops were held in the months following the field manuals release (July - September 2018), including focused workshops on bathymetry data, marine imagery, and biological specimen data. The bathymetry data release protocols will depend on new digital infrastructure being scoped as part of the AusSeabed program ${ }^{8}$. In contrast, marine imagery and biological specimen data are linked to the existing platforms mentioned above so priorities are to establish appropriate workflows linking these platforms with the data collection phase, and to find the resources needed to ensure they can be developed and maintained.

\section{Ensuring Uptake}

The uptake of the SOPs described by the field manuals was a concern for two reasons. Firstly, agency-specific sampling protocols are integral to several ongoing monitoring programs (e.g., the Long-Term Monitoring Program of the Australian Institute of Marine Science in De'ath et al., 2012), and attempts

\footnotetext{
${ }^{3}$ www.squidle.org

${ }^{4}$ www.globalarchive.org

${ }^{5}$ https://benthobox.com

${ }^{6}$ www.ala.org.au

${ }^{7}$ www.obis.org.au

${ }^{8}$ www.ausseabed.gov.au
}

to alter or change methods associated with these may jeopardize data comparability. Secondly, switching to a new protocol may be costly and laborious, making individuals or agencies unwilling or unable to adapt. These challenges meant the field manuals had to balance being overly prescriptive (such that people prefer to follow their own protocol and ignore the manuals) and overly flexible (such that data are not consistent and therefore not comparable). A collaborative approach to developing the manuals was therefore paramount, and to that end, representatives from agencies involved in ongoing monitoring programs, including marine managers from all Australian states and territories, were engaged. This ensured that methods could accommodate existing programs and differences were clearly explained in relation to marine monitoring in state and Commonwealth waters.

Nonetheless, challenges remain with promoting uptake in certain sectors, namely private consultancies and the petroleum industry. These sectors are often strongly linked, with consultants engaged by industry to undertake various environmental characterisations and impact assessments, each of which uses their own methods (Gray et al., 1999). We accentuated the commercial value to industry in adopting standards, including access to larger spatial and temporal range of comparable data to provide regional context to potentially impacted locations, and this has been promoted by the national offshore petroleum regulator (NOPSEMA, 2018). However, this premise depends on the sharing of data (e.g., Marine Data Exchange in ocean renewable industry). This has proven challenging to implement among the petroleum industry in Australia, although there have been some efforts for meta-data sharing through the Industry-government Environmental Meta-database (IGEM) by the Western Australian Marine Science Institution.

Field manuals were released and promoted on the Marine Biodiversity $\mathrm{Hub}^{9}$, an Australian Government-funded collaboration of marine research institutions with a mandate to improve provision of marine biodiversity information nationally. During release and promotions, we emphasized that the current package of field manuals is not meant to immediately replace most existing protocols, but rather to complement them for national monitoring purposes. At the same time, we hope that marine monitoring programs will also identify opportunities to adjust current practices to increase national consistency, and that the SOPs will provide an opportunity for industry and industry consultants to contribute to national monitoring through standardizing their ongoing activities.

\section{Promoting International Relevance}

The SOPs were developed for use in Australia's Exclusive Economic Zone, but due to the diversity of habitats and communities included in this area, they are applicable to other oceanic regions. The SOPs have been made available internationally through the Ocean Best Practice portal ${ }^{10}$; however, it is clear that further work will be required to promote international uptake. Discussions have been held

\footnotetext{
${ }^{9}$ www.nespmarine.edu.au

${ }^{10}$ www.oceanbestpractices.org
} 
with the IOC-UNESCO Global Ocean Observing System on how these SOPs can support global monitoring through the Essential Ocean Variables (Miloslavich et al., 2018), and they have been made available to the Group on Earth Observations Biodiversity Observation Networks' "Biodiversity Observation in a BOX" (boninabox.geobon.org). Further coordination of the global marine community is essential if the results of monitoring are to have the impact that is needed to inform the diverse set of international conventions, agreements and products including (to name just 4 out of more than 20), the Convention on Biological Diversity's Aichi Targets, the United Nations (UN) Sustainable Development Goals, the second UN World Ocean Assessment, and the Intergovernmental SciencePolicy Platform on Biodiversity and Ecosystem Services regional assessments. At present, many of these agreements rely on indicators derived from highly aggregated data that poorly reflects management outcomes This is a symptom of both a lack of monitoring capacity, especially in the developing countries, but also the lack of marine scientists working together globally to emphasize their common message. SOPs are one way to start increasing the relevance of marine science to the global decisionmaking community.

\section{FUTURE DIRECTIONS}

For the potential of these field manuals to be fully realized, they must be maintained, updated, and their use and impact measured. In the immediate future, version 2 of all manuals will be released in 2019. At the moment, potential users should contact the lead author of this paper for further information, but in the next version of manuals we anticipate that a general contact point or help desk will be created. Review cycles beyond the development of Version 2 have yet to be developed. A plan for long-term maintenance will ensure that the field manuals remain accessible and topical. The latter point is particularly applicable to sampling platforms that are technologically complex (e.g., autonomous underwater vehicles) or new, but even platforms that have been established for decades (e.g., sleds, trawls, grabs, and box corers) may require periodic updates related to data release as digital infrastructure continues to evolve and our understanding of new areas such as microplastics, the microbial community or e-DNA become sufficiently mature for regular monitoring. Major updates must be documented in each new version with appropriate version control. One of the main actions needed to ensure longevity of the field manuals is the establishment of an oversight committee that can provide longterm and multi-institutional support. This responsibility will be further explored with Australia's National Marine Science Committee and its associated working groups.

Measuring the impact of SOPs can help inform future versions, identify gaps or problems, and ensure future support and funding. In recognition of the importance of this task, the original Marine Biodiversity Hub project has been extended with a milestone focused on assessing uptake and impact. Formal impact assessment frameworks such as CSIRO's Impact Evaluation Guide (CSIRO, 2015) will be scoped, as well as informal assessments stemming from an online questionnaire and periodic literature searches.

We anticipate that the national standards for marine sampling developed here may either be adapted for use in countries without national SOPs or mapped to SOPs in countries that have already developed them (e.g., Joint Nature Conservation Committee in Burns et al., 2018), thus contributing to globally consistent datasets. The ocean observing community is rapidly moving toward globally consistent standards (Bax et al., 2018), but the biological community and the marine benthic sampling community in particular have been constrained to regional or national efforts. This is due to the fine-scale biogeography of many benthic communities and the need for tailored sampling and analyses among different habitats, as well as the technological capacity and scientific development in different countries. SOPs are urgently needed to increase the impact of marine science on global policy and decisions. The UN Decade of Ocean Science for Sustainable Development includes improved marine monitoring as one of its key objectives to support Agenda 2030 (Visbeck, 2018). We hope that the process of SOP development described in the current paper, as well as the SOPs themselves, can provide the start of a discussion that marine biologists need to have to maximize our impact in the next decade.

\section{AUTHOR CONTRIBUTIONS}

All authors lead or significantly contributed to the development of one or more of the of the field manuals upon which this manuscript is based. All authors contributed to the writing of the manuscript.

\section{ACKNOWLEDGMENTS}

This field manual package would not have been possible without the expertise of our many collaborators listed in Appendix A: www.nespmarine.edu.au/sites/default/files/_PUBLIC_/Field Manuals_NESPMarineHub_Appendices_v1.pdf. This work was undertaken for the Marine Biodiversity Hub, a collaborative partnership supported through funding from the Australian Government's National Environmental Science Program (NESP). NESP Marine Biodiversity Hub partners include the University of Tasmania; CSIRO, Geoscience Australia, Australian Institute of Marine Science, Museum Victoria, Charles Darwin University, The University of Western Australia, Integrated Marine Observing System, NSW Office of Environment and Heritage, NSW Department of Primary Industries. Scott Nichol and several reviewers provided valuable comments on this paper. RP and AC publish with the permission of the CEO, Geoscience Australia.

\section{SUPPLEMENTARY MATERIAL}

The Supplementary Material for this article can be found online at: https://www.frontiersin.org/articles/10.3389/fmars. 2019.00177/full\#supplementary-material 


\section{REFERENCES}

Albert, C. H., Yoccoz, N. G., Edwards, T. C., Graham, C. H., Zimmermann, N. E., and Thuiller, W. (2010). Sampling in ecology and evolution - bridging the gap between theory and practice. Ecography 33, 1028-1037. doi: 10.1111/j.16000587.2010.06421.x

Althaus, F., Hill, N., Ferrari, R., Edwards, L., Przeslawski, R., Schönberg, C. H. L., et al. (2015). A standardised vocabulary for identifying benthic biota and substrata from underwater imagery: the CATAMI classification scheme. PLoS One 10:e0141039. doi: 10.1371/journal.pone.0141039

Bates, A. E., Bird, T. J., Stuart-Smith, R. D., Wernberg, T., Sunday, J. M., Barrett, N. S., et al. (2015). Distinguishing geographical range shifts from artefacts of detectability and sampling effort. Divers. Distrib. 21, 13-22. doi: 10.1111/ddi. 12263

Bax, N. J., Appeltans, W., Brainard, R., Duffy, J. E., Dunstan, P., Hanich, Q., et al. (2018). Linking capacity development to GOOS monitoring networks to achieve sustained ocean observation. Front. Mar. Sci. 5:356. doi: 10.3389/fmars. 2018.00346

Boss, E., Waite, A., Muller-Karger, F., Yamazaki, H., Wanninkhof, R., Uitz, J., et al. (2018). Beyond chlorophyll fluorescence: the time is right to expand biological measurements in ocean observing programs. Limnol. Oceanogr. Bull. 27, 89-90. doi: 10.1002/lob.10243

Bouchet, P., Huang, Z., Phillips, C., Meeuwig, J., Foster, S., and Przeslawski, R. (2018). Comparative Assessment of Pelagic Sampling Platforms. Perth, WA: University of Western Australia.

Burns, F., Eaton, M. A., Hayhow, D. B., Outhwaite, C. L., Al Fulaij, N., August, T. A., et al. (2018). An assessment of the state of nature in the United Kingdom: a review of findings, methods and impact. Ecol. Indic. 94, 226-236. doi: 10.1016/ j.ecolind.2018.06.033

Butler, A. J., Rees, T., Beesley, P., and Bax, N. J. (2010). Marine biodiversity in the australian region. PLoS One 5:e11831. doi: 10.1371/journal.pone.0011831

Cochrane, P. (2016). The marine protected area estate in Australian (Commonwealth) waters, in: Fitzsimons, J., Westcott, G. (Eds.), Big Bold and Blue. Melbourne, VIC: CSIRO Publishing, 45-63.

Coro, G., MAgliozzi, C., Berghe, E. V., Bailly, N., Ellenbroek, A., and Pagano, P. (2016). Estimating absence locations of marine species from data of scientific surveys in OBIS. Ecol. Model. 323, 61-76. doi: 10.1016/j.ecolmodel.2015.12.008

Costello, M. J., Basher, Z., McLeod, L., Asaad, I., Claus, S., Vandepitte, L., et al. (2017). Methods for the Study of Marine Biodiversity, in: Walters, M., Scholes, R. J. (Eds.), The GEO Handbook on Biodiversity Observation Networks (Cham: Springer International Publishing) 129-163. doi: 10.1007/978-3-31927288-7_6

CSIRO (2015). Impact Evaluation Guide. Canberra, ACT: CSIRO.

De'ath, G., Fabricius, K. E., Sweatman, H., and Puotinen, M. (2012). The 27-year decline of coral cover on the great barrier reef and its causes. Proc. Natl. Acad. Sci. U.S.A. 109, 17995-17999. doi: 10.1073/pnas.1208909109

Duffy, J. E., Amaral-Zettler, L. A., Fautin, D. G., Paulay, G., Rynearson, T. A., Sosik, H. M., et al. (2013). Envisioning a marine biodiversity observation network. BioScience 63, 350-361. doi: 10.1525/bio.2013.63.5.8

Dunstan, P. K., and Dambacher, J. (2019). Options for Assessing Cumulative Impact and Risk to Environmental Values in Matters of National Environmental Significance and Australian Marine Parks, Report to the National Environmental Science Programme, Marine Biodiversity Hub. Hobart, TAS: CSIRO.

Fancy, S. G., Gross, J. E., and Carter, S. L. (2009). Monitoring the condition of natural resources in US national parks. Environ. Monit. Assess. 151, 161-174. doi: 10.1007/s10661-008-0257-y

GBRMPA (2015). Reef 2050 Integrated Monitoring and Reporting Program Strategy. Townsville: Great Barrier Reef Marine Park Authority \& Queensland Government.

Goddard, J. H. R., and Foster, N. R. (2002). Range extensions of sacoglossan and nudibranch molluscs (Gastropoda: Opisthobrania) to Alaska. Veliger 45, 331-336.
Gray, J. S., Bakke, T., Beck, H. J., and Nilssen, I. (1999). Managing the environmental effects of the norwegian oil and gas industry: from conflict to consensus. Mar. Pollut. Bull. 38, 525-530. doi: 10.1016/S0025-326X(99)00004-1

Hoenner, X., Huveneers, C., Steckenreuter, A., Simpfendorfer, C., Tattersall, K., Jaine, F., et al. (2018). Australia’s continental-scale acoustic tracking database and its automated quality control process. Sci. Data 5:170206. doi: 10.1038/ sdata.2017.206

Lara-Lopez, A., Moltmann, T., Proctor, R., 2016. Australia’s integrated marine observing system (IMOS): data impacts and lessons learned. Mar. Technol. Soc. J. 50, 23-33. doi: 10.4031/MTSJ.50.3.1

Miloslavich, P., Bax, N. J., Simmons, S. E., Klein, E., Appeltans, W., AburtoOropeza, O., et al. (2018). Essential ocean variables for global sustained observations of biodiversity and ecosystem changes. Glob. Change Biol. 24, 2416-2433. doi: $10.1111 / \mathrm{gcb} .14108$

Monk, J. (2014). How long should we ignore imperfect detection of species in the marine environment when modelling their distribution? Fish Fish. 15, 352-358. doi: 10.1111/faf.12039

NOPSEMA (2018). Sharing Knowledge about the Marine Environment. Perth, WA: NOPSEMA.

Oldekop, J. A., Fontana, L. B., Grugel, J., Roughton, N., Adu-Ampong, E. A., Bird, G. K., et al. (2016). 100 key research questions for the post-2015 development agenda. Dev. Policy Rev. 34, 55-82. doi: 10.1111/dpr.12147

Przeslawski, R., and Foster, S. (eds) (2018). Field Manuals for Marine Sampling to Monitor Australian Waters, Version 1. 2018, (Canberra, ACT: National Environment Science Programme Marine Biodiversity Hub), 212. doi: $10.11636 / 9781925297669$

Przeslawski, R., Brooke, B., Carroll, A. G., and Fellows, M. (2018a). An integrated approach to assessing marine seismic impacts: lessons learnt from the gippsland marine environmental monitoring project. Ocean Coast. Manag. 160, 117-123. doi: 10.1016/j.ocecoaman.2018.04.011

Przeslawski, R., Foster, S., Monk, J., Langlois, T. J., Lucieer, V. L., and Stuart-Smith, R. D. (2018b). Comparative Assessment of Seafloor Sampling Platforms. Mumbai: National Environmental Science Programme, 57.

Stuart-Smith, R. D., Edgar, G. J., Barrett, N. S., Bates, A. E., Baker, S. C., Bax, N. J., et al. (2017). Assessing national biodiversity trends for rocky and coral reefs through the integration of citizen science and scientific monitoring programs. BioScience 67, 134-146. doi: 10.1093/biosci/biw180

Teixeira, H., Berg, T., Uusitalo, L., Fürhaupter, K., Heiskanen, A. -S., Mazik, K., et al. (2016). A catalogue of marine biodiversity indicators. Front. Mar. Sci. 3:207. doi: $10.3389 /$ fmars.2016.00207.

Visbeck, M. (2018). Ocean science research is key for a sustainable future. Nat. Commun. 9:690. doi: 10.1038/s41467-018-03158-3

Wilkinson, M. D., Dumontier, M., Aalbersberg, I. J., Appleton, G., Axton, M., Baak, A., et al. (2016). The FAIR guiding principles for scientific data management and stewardship. Sci. Data 3:160018. doi: 10.1038/sdata.2016.18

Woodall, L. C., Andradi-Brown, D. A., Brierley, A. S., Clark, M. R., Connelly, D., Hall, R. A., et al. (2018). A multidisciplinary approach for generating globally consistent data on mesophotic, deep-pelagic, and bathyal biological communities. Oceanography 31, 76-89. doi: 10.5670/oceanog. 2018.301

Conflict of Interest Statement: The authors declare that the research was conducted in the absence of any commercial or financial relationships that could be construed as a potential conflict of interest.

Copyright (C) 2019 Przeslawski, Foster, Monk, Barrett, Bouchet, Carroll, Langlois, Lucieer, Williams and Bax. This is an open-access article distributed under the terms of the Creative Commons Attribution License (CC BY). The use, distribution or reproduction in other forums is permitted, provided the original author(s) and the copyright owner(s) are credited and that the original publication in this journal is cited, in accordance with accepted academic practice. No use, distribution or reproduction is permitted which does not comply with these terms. 